\title{
A natural odor attraction between lactic acid bacteria and the nematode Caenorhabditis elegans
}

\author{
Jae Im Choi, Kyoung-hye Yoon, Saraswathi Subbammal Kalichamy, Sung-Sik Yoon \\ and Jin Il Lee \\ Division of Biological Science and Technology, College of Science and Technology, Yonsei University, \\ Wonju, Korea
}

\begin{abstract}
Animal predators can track prey using their keen sense of smell. The bacteriovorous nematode Caenorhabditis elegans employs sensitive olfactory sensory neurons that express vertebrate-like odor receptors to locate bacteria. C. elegans displays odor-related behaviors such as attraction, aversion and adaptation, but the ecological significance of these behaviors is not known. Using a combination of food microbiology and genetics, we elucidate a possible predator-prey relationship between $C$. elegans and lactic acid bacteria (LAB) in rotting citrus fruit. LAB produces the volatile odor diacetyl as an oxidized by-product of fermentation in the presence of citrate. We show that $C$. elegans is attracted to LAB when grown on citrate media or Citrus medica $L$, commonly known as yuzu, a citrus fruit native to East Asia, and this attraction is mediated by the diacetyl odor receptor, ODR-10. We isolated a wild LAB strain and a wild $C$. elegans-related nematode from rotten yuzu, and demonstrate that the wild nematode was attracted to the diacetyl produced by LAB. These results not only identify an ecological function for a $C$. elegans olfactory behavior, but contribute to the growing understanding of ecological relationships between the microbial and metazoan worlds.
\end{abstract}

The ISME Journal (2016) 10, 558-567; doi:10.1038/ismej.2015.134; published online 4 August 2015

\section{Introduction}

For decades, field biologists have elegantly described behaviors that arise due to ecological pressures. However, with the onset of the genome era, scientists are beginning to look at ecology through a molecular lens, focusing on the role of genes and proteins in producing ecologically relevant behaviors. This has led to laboratory-based experiments that have begun to elucidate molecular mechanisms of behavioral ecology. For instance, specific receptors for both pheromones and odors were shown to be important for mice to identify and evade predators such as cats, rats and mountain lions (Papes et al., 2010; Ferrero et al., 2011).

Caenorhabditis elegans, a free-living soil nematode, has been used in the laboratory as a genetic model organism for decades. C. elegans displays many robust behaviors related to sex, temperature and food searching (Hedgecock and Russell, 1975; Loer and Kenyon, 1993; Liu and Sternberg, 1995; Hills et al., 2004; Gray et al., 2005). In addition, this

Correspondence: S-S Yoon or J Il Lee, Division of Biological Science and Technology, College of Science and Technology, Yonsei University, 1 Yonseidae-gil, Wonju, Gangwondo 220-710, Korea.

E-mail: sungsik@yonsei.ac.kr or jinillee@yonsei.ac.kr

Received 21 March 2015; revised 29 May 2015; accepted 9 June 2015; published online 4 August 2015 nematode can sense dozens of odors through several olfactory sensory neurons (Bargmann et al., 1993), and shows attraction to, aversion and memory of many of these odors (Ward, 1973; Dusenbery, 1975; Bargmann and Horvitz, 1991; Colbert and Bargmann, 1995). Odors are detected through G-protein coupled odor receptors (GPCRs) similar to receptors in mammals (Buck and Axel, 1991; Bargmann et al., 1993). For instance, the odor diacetyl is sensed by the ODR-10 receptor (Sengupta et al., 1996), one of hundreds of G-protein coupled odor receptorencoding genes that are scattered throughout the C. elegans genome (Robertson, 1998).

Although a plethora of studies have shown the neurons, circuitry and molecules responsible for odor behaviors, the ecological significance of these behaviors in nature are not known. In the laboratory, C. elegans is maintained on agar plates and feed on Escherichia coli, a bacteria normally found in the intestine of animals, and rarely encountered in nature. In the wild, C. elegans can be found in the soil throughout the world, but recent studies have shown that they are gathered in large numbers in rotten fruit and vegetation (Barriere and Felix, 2005; Felix and Braendle, 2010). The exact reason for this is not fully known; however, rotten fruit and vegetation are a rich source of bacteria for worms to feed on. Rhabditid nematodes like C. elegans are considered enrichment-type nematodes 
(Bongers, 1990; Darby et al., 2007). They normally exist in a semi-hibernating dauer larval stage in nature (Barriere and Felix, 2005), but when they come upon a rich source of bacteria, the dauers will quickly grow to sexually mature adults, rapidly reproduce in very large numbers, and consume much of the bacteria present (Bongers, 1990). Since fruits are a rich source of sugar and nutrients for bacteria, large bacterial bonanzas of many different species grow and ferment the fruit over time. If $C$. elegans can sense and track rotting fruit, they will likely find bacterial bonanzas blooming throughout, allowing the nematodes to reproduce and flourish.

Lactic acid bacteria (LAB) are a clade of grampositive bacteria that produce lactic acid as an end product of carbohydrate fermentation. They are found in fermenting vegetative matter and milk, and are major contributors to the production and flavoring of fermented foods such as yogurts and cheeses (Marshall, 1987; Smit et al., 2005). LAB also are found in the human gut, and are associated with positive effects to health (Guarner and Malagelada, 2003; Hempel et al., 2012). Hence, many LAB are also considered to be probiotic. LAB can survive well in low-pH environments such as fruit, which is a tremendous source of sugar and nutrients to growing bacterial populations (Trias et al., 2008). One of the fermentative strategies that LAB has at its disposal when grown on fruit is to utilize citrate together with sugar (Hugenholtz, 1993). A by-product of this fermentative pathway is the production of the volatile chemical diacetyl (butane2,3-dione) (Hammer, 1920; Michaelian et al., 1933, 1938), which emits a buttery odor that is often used commercially in popcorn.

A few studies have explored the relationship between $C$. elegans and LAB in the laboratory. Exposure to LAB can induce the expression of genes involved in innate immunity providing protection for C. elegans particularly towards gram-positive bacterial infection (Ikeda et al., 2007; Kim and Mylonakis, 2012). However, little is understood about the ecological relationship between nematodes and LAB in nature. As C. elegans can be found in rotting fruit and has a strong attraction for diacetyl (Bargmann et al., 1993), one possibility is that production of diacetyl from LAB growing on fruit may be one of the cues that nematodes use to find bacterial bonanzas. In this study, we show that LAB grown on citrate media or citrus fruit media produces diacetyl, and $C$. elegans is attracted to diacetyl produced by LAB via the ODR-10 diacetyl receptor. We tested $\mathrm{LAB}$ and rhabditid nematodes isolated from citrus fruit, and showed that these nematodes are naturally attracted to diacetylproducing $\mathrm{LAB}$. We speculate that the by-product diacetyl produced from LAB grown on citrate is an environmentally relevant cue that allows nematodes to track bacterial bonanzas located in citrus fruit.

\section{Materials and methods}

\section{Strains and maintenance}

All C. elegans strains were maintained at $20^{\circ} \mathrm{C}$ on nematode growth medium (NGM) seeded with OP50 strain E. coli (Lewis and Fleming, 1995). Bristol N2 wild type and CX32 odr-10(ky32) were obtained from the Caenorhabditis Genetics Center. Lactobacillus paracasei strain YSM0385 was isolated from yogurt in the Yoon lab (Song et al., 2013). LAB was grown at $37^{\circ} \mathrm{C}$ on Difco Lactobacillus MRS Broth (BD, Sparks, MD, USA, Cat\# 288130) before inoculating on to different agar media plates used in the study.

\section{Collection and identification of wild nematode}

Collection of soil+rotten fruit samples occurred at the following locations: Se Wook Farms (Wonju), Chiaksan Peach Farms (Wonju), Ja So Dahm Farms (Goheung) and a private farm in Chungju. Wild nematode strains were isolated and identified following published methods (Barrière and Félix, 2014). Briefly, a small portion of the samples were placed on the edge of NGM plates with an E. coli OP50 lawn in the middle. Individual or a group of worms were picked into separated seeded NGM plates. Worms were first categorized by morphology, and several worms from each category were sequenced. Sequences from $18 \mathrm{~S}$ rDNA and ITS2 region were PCR amplified and compared against known sequences using NCBI BLAST or the Rhabditina DB database (http://128.122.60.136/fmi/iwp/ cgi?-db = RhabditinaDB\&-loadframes) to identify the nematode strains. Primers used were: SSU18A (5'-AAAGATTAAGCCATGCATG) and SSU26R (5'-CATTCTTGGCAAATGCTTTCG) for $18 \mathrm{~S}$ rDNA sequence amplification (Floyd et al., 2002), and 5.8 S-1 (5'-CTGCGTTACTTACCACGAATTGCARAC) and KK28S-4 (5'-GCGGTATTTGCTACTACCAYYA MGATCTGC) for amplifying the ITS2 region (Kiontke et al., 2011).

\section{Collection and identification of wild $L A B$}

To isolate wild LAB strains from the soil+fruit sample described above, a portion of each sample was mixed with water, vortexed, then centrifuged. The supernatant was serially diluted to $10^{-4}$ and then to $10^{-6}$, and plated on MRS agar plates containing sodium azide and bromocresol purple (Yakuri, Japan) (0.05 g of each in $1 \mathrm{l}$ media). Sodium azide inhibits growth of gram-negative bacteria, and bromocresol purple is a $\mathrm{pH}$ indicator that allows identification of LAB colonies by the color change of the surrounding agar from purple to yellow, due to lactic acid production. For molecular identification of LAB isolates, a fragment of $16 \mathrm{~S}$ rDNA sequence was PCR amplified using the following primers: $27 \mathrm{~F}$ forward primer (5'-AGAGTTTGATCMTGGCTCAG) and 1492 reverse primer (5'-TACGGYTACCTTGTT ACGACTT). 
Bacterial culture and media

Culture media was prepared similarly to a previous study (Jyoti et al., 2004). The composition of $1 \mathrm{~L}$ of minimal media (M), which is a modified MRS, is as follows: $5 \mathrm{~g}$ yeast extract (BD, Cat\#212750) $0.1 \mathrm{~g}$ $\mathrm{MgSO}_{4} \cdot 7 \mathrm{H}_{2} \mathrm{O}, 0.05 \mathrm{~g} \mathrm{MnSO} \cdot \mathrm{H}_{2} \mathrm{O}, 2 \mathrm{~g} \mathrm{NaH} \mathrm{PO}_{4}, 1 \mathrm{ml}$ Tween80, $15 \mathrm{~g}$ agar. When supplementing glucose, $6.3 \mathrm{~g}$ of glucose was added to the minimal media, and for supplementing citrate, $2.2 \mathrm{~g}$ of sodium citrate was used according to previous protocols (Jyoti et al., 2004). For fruit media, fresh fruit either directly from the above farms or store-bought was used for yuzu and apple media. For tomato media agar, organic tomato paste (Costco, Seattle, WA, USA, Item 633564) was used. For barley malt media agar, barley malt (BD, Cat\#218630) was used. Whole yuzu fruits, apples or tomato paste were pureed with equal volumes of distilled water in a kitchen blender, and centrifuged at 8500 r.p.m. in a high speed centrifuge (Hanil Supra $30 \mathrm{~K}$, Korea) in $500 \mathrm{ml}$ bottles. The supernatant was buffered to $\mathrm{pH}$ 6.5 for optimal LAB growth and $2 \%$ agar was added and then autoclaved at $121{ }^{\circ} \mathrm{C}$ for $5 \mathrm{~min}$. The agar media was then poured into plates. For barley malt media, $1.5 \%$ agar was added to barley malt and autoclaved.

\section{Behavior assays}

Odortaxis assays were performed on $9 \mathrm{~cm}$ petri dishes containing 1.6\% Difco granulated agar (BD, Cat\#214530) similar to the previously published methods (Bargmann et al., 1993) except with some adjustments. A 7.5-mm agar plug from a media plate was made by using the back end of a 1000- $\mu \mathrm{l}$ disposable micropipette tip. An agar plug without bacteria was placed on one side of the lid of a 9-cm petri dish. An agar plug of the same type of media plate with bacteria was placed on the other side of the same lid. Two microliters of $1 \mathrm{M}$ sodium azide was placed directly below the agar plugs on the surface of the plate to paralyze the worms upon reaching the area of the plate directly below the agar plug. A population of adult worms were placed onto the origin of the assay plate and allowed to move freely on the plate for an hour. For chemical odor assays, $2 \mu \mathrm{l}$ of diacetyl (Sigma, St Louis, MO, USA, 1:1000 dilution in ethanol) and $2 \mu \mathrm{l}$ of benzaldehyde (Sigma, 1:200 dilution in ethanol) was used. A 2.5-cm square box surrounding each spot of diacetyl was drawn, and attraction index was calculated as ((\# of worms at the odor) - (\# of worms at the control side))/(total \# of worms).

For odor adaptation assays, methods similar to previous methods (Colbert and Bargmann, 1995) were used. Briefly, populations of adult worms were incubated in either a solution of 1:5000 dilution of diacetyl or 1:5000 dilution of benzaldehyde in S-basal buffer for $80 \mathrm{~min}$. Worms were washed three times in S-basal, and subjected to the attraction assays described above.

\section{Bacteria colony counts}

An agar plug containing bacteria after 2 days of growth at $37^{\circ} \mathrm{C}$ from each of the media was placed in $0.85 \% \mathrm{NaCl}$, and then diluted to $10^{-5}, 10^{-6}$ and $10^{-7}$. One-hundred microliters of these dilutions were plated on a 9-cm MRS agar plate overnight in duplicate, then counted. The trials were repeated on three separate days for Min and Min+Citr, four separate days for Min+Glu, five separate days for MRS and six separate days for Min+Glu+Citr.

\section{Quantification of diacetyl}

Concentration of diacetyl was quantified by modifying a spectrophotometric assay previously described (Mattessich and Cooper, 1989). Briefly, a solution containing $325 \mu \mathrm{l}$ of solution A (saturated solution of creatine), $150 \mu \mathrm{l}$ of solution B $(3 \% \mathrm{NaOH}, 3.5 \%$ $\alpha$-naphthol) and $275 \mu \mathrm{l}$ of distilled water was prepared, to which an agar plug with LAB was added and vortexed to release diacetyl that may have seeped into the agar. The mixture was spun down at 10,000 r.p.m. for $1 \mathrm{~min}$ and the supernatant was passed through a $0.25-\mu \mathrm{m}$ membrane filter (Advantec, Toyo Roshi Kaisha, Japan) to eliminate any precipitates that may have formed. The reaction was left to develop for $1 \mathrm{~h}$, and $100 \mu \mathrm{l}$ of the solution was each added to three wells in a 96-well plate. Thus, each sample was performed in triplicate in each experiment. Absorbance at $525 \mathrm{~nm}$ was measured using a spectrophotometric plate reader (Infinite M200Pro, Tecan, Switzerland). Fresh solution B was prepared for each assay. Using commercially available diacetyl, we produced standard curves for diacetyl concentration (Supplementary Figure S2), and then measured the concentration of diacetyl produced by LAB from the agar plug of various types of media. Each data point from each experiment is an average of the triplicate samples.

\section{Results}

\section{L. paracasei produces diacetyl in the presence} of citrate

It is well known in the food industry that LAB strains will produce the volatile chemical diacetyl when grown on media containing citrate (Michaelian et al., 1933; Figure 1b). Citrate provides an extra source of pyruvate for lactic acid producing bacteria, and shifts sugar metabolism to allow the production of the buttery odor diacetyl as an oxidized by-product (Hugenholtz, 1993). Food producers use this ability of LAB to produce diacetyl to flavor fermented foods such as cheeses, yogurts and wine (Marshall, 1987; Bartowsky and Henschke, 2004; Smit et al., 2005). As C. elegans is attracted to dozens of odors tested in the laboratory including diacetyl (Bargmann et al., 1993), we asked whether diacetyl produced by LAB can be sensed and induce attraction behavior in C. elegans. We chose to use a strain of Lactobacillus paracasei, which can be found in plants, many dairy 
a

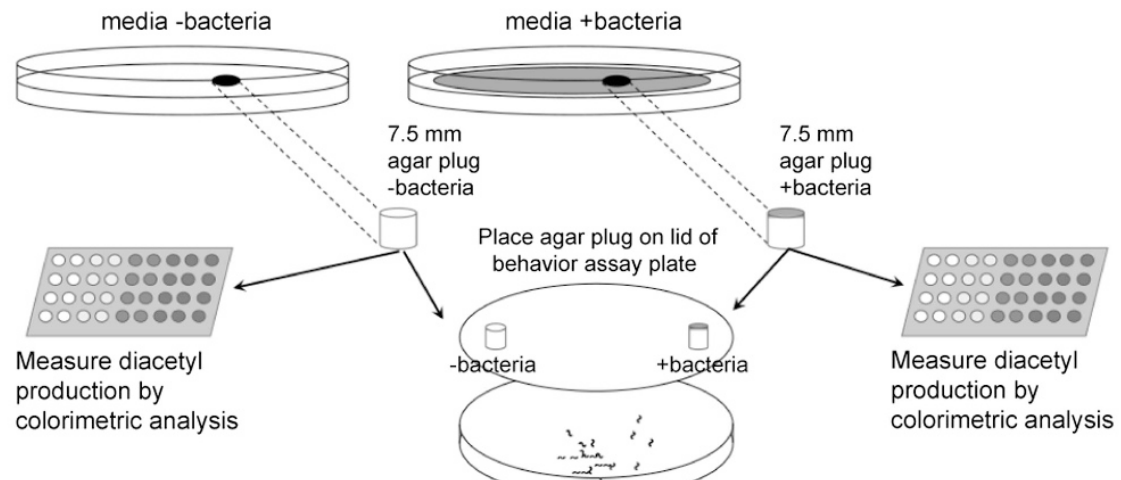

Measure $C$. elegans attraction behavior

b

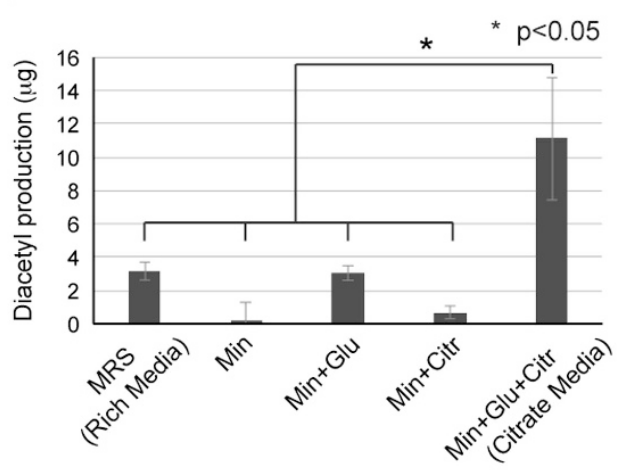

C

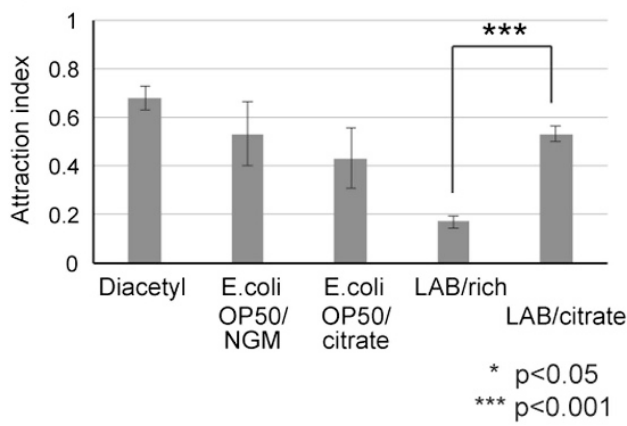

d
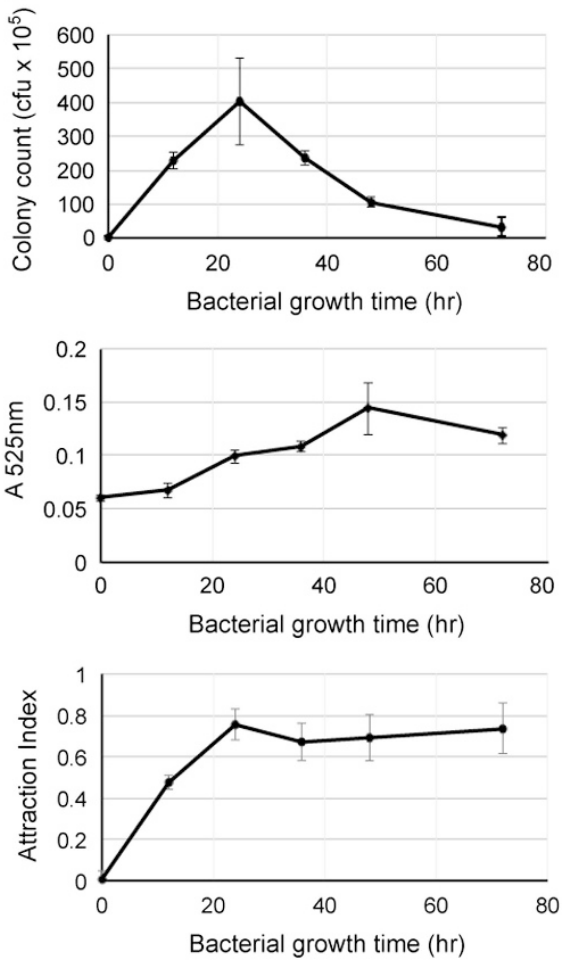

Figure 1 C. elegans is attracted to lactic acid bacteria grown on media containing citrate. (a) Strategy of study. L. paracasei (LAB) or E. coli strain OP50 is grown on several types of media (media +bacteria) including a rich MRS media (rich), a minimal media (min), minimal media containing glucose (min+glu), minimal media containing citrate (min+citr), minimal media containing both glucose and citrate (min+glu+citr) and nematode growth media (NGM). Media without bacteria (media-bacteria) is used as a control. A 7.5-mm agar plug is taken from each plate and used either for colorimeteric analysis to measure diacetyl production, or attraction behavior assay. In the attraction behavior assay, the +bacteria agar plug is placed on the lid of a 9-cm plate, and the -bacteria agar plug is placed on the opposite side, and C. elegans are allowed to move freely on an agar assay plate (see Materials and Methods). (b) Diacetyl production of L. paracasei grown for 2 days on different types of media. Diacetyl concentration is measured by colorimetric analysis in four separate experiments (Diacetyl standard curves are provided in Supplementary Figure S2). Error bars indicate s.e. (c) C. elegans attraction behavior to diacetyl (1:1000 dilution), E. coli strain OP50 or L. paracasei (LAB) grown for 2 days on rich or citrate media. Error bars indicate s.e. (d) Bacterial colony count (top), diacetyl production (middle) and C. elegans attraction behavior (bottom) to LAB grown on citrate media over $72 \mathrm{~h}$. Colony count is measured as colony forming units (cfu). Diacetyl levels are measured as sample absorbance at $525 \mathrm{~nm}$. Error bars indicate s.e. Significance was calculated by Student's $t$-test and $P$-values are indicated.

products and as a commensal in the human gut. It is also commonly used as a probiotic and is known to produce diacetyl (Aunsbjerg et al., 2015). For this study we use L. paracasei and the term 'LAB' interchangeably.
First, we tested the ability of $L$. paracasei to produce diacetyl when grown in different nutrient conditions. We grew LAB for $48 \mathrm{~h}$ on several types of agar media: a rich MRS media, a minimal (M) media alone, or a minimal media added with either glucose, 
citrate or glucose and citrate (Figure 1b). Then we took a 7.5-mm cylindrical agar plug from media plates with or without bacteria, and measured diacetyl levels by using a previously published spectrophotometric method for diacetyl concentration (Figure 1a) (Mattessich and Cooper, 1989). We found that LAB grown on rich media produced $\sim 3 \mu \mathrm{g}$ of diacetyl, whereas LAB grown on minimal media produced very little (Figure 1b). Supplementing the minimal media with citrate alone had little effect in diacetyl production, whereas supplementing with glucose increased production to about $3 \mu \mathrm{g}$. However, when the media was supplemented with both glucose and citrate, there was a 15-fold increase in diacetyl production compared with citrate alone, and a 3.5-fold increase compared with glucose alone or rich media. This was not due to differential growth and the number of bacteria in the different media, as there seemed to be very little correlation between diacetyl production and bacteria counts (Supplementary Figure S1). For example, although the average cell counts of LAB in the rich media and the minimal media supplemented with glucose and citrate were similar, diacetyl production was 3.5-fold higher in the latter (Supplementary Figure S1 and Figure 1b). Thus, LAB growth in the presence of both glucose and citrate (hereby referred to as 'citrate media') leads to a substantial production of diacetyl.

\section{C. elegans is attracted to L. paracasei grown on citrate} media

We next asked whether $C$. elegans is more attracted to LAB that produce diacetyl. We modulated previously published methods (Ward, 1973; Bargmann et al., 1993) to assess C. elegans attraction to $7.5 \mathrm{~mm}$ agar plugs with or without bacteria for each type of media. As shown previously (Bargmann et al., 1993; Sengupta et al., 1996), worms showed strong attraction to commercially available chemical diacetyl and OP50 strain E. coli, the strain of bacteria that worms are normally cultured on in the laboratory (Figure 1c). When we tested odor attraction to bacteria, adult worms that were cultivated on NGM seeded with OP50 naturally were attracted to their familiar culture conditions, but also moderately attracted to OP50 when grown in citrate media. $\mathrm{LAB}$, on the other hand, was minimally attractive when grown in rich media. Interestingly, however, when LAB was grown on citrate media, worms showed a strong attraction similar to OP50 strain E. coli grown on NGM. Thus, C. elegans can sense a difference in $L$. paracasei grown in the presence of citrate.

It is no surprise that $C$. elegans can smell bacteria (Harris et al., 2014), and one possibility is that worms are simply sensing a large growth of LAB on citrate. Although we showed that LAB cell counts in the rich media and the citrate media did not correlate with their respective attraction index to worms (Supplementary Figure S1), we investigated this possibility further. We tracked LAB growth on citrate media for $72 \mathrm{~h}$, and measured bacterial growth, diacetyl production, and $C$. elegans attraction behavior (Figure 1d). LAB growth on citrate media peaked at $24 \mathrm{~h}$, decreased considerably by $48 \mathrm{~h}$ and by $72 \mathrm{~h}$ most of the bacteria had died (Figure 1d, top). On the other hand, diacetyl production and accumulation continued for $48 \mathrm{~h}$, and levels remained high even after $72 \mathrm{~h}$ (Figure 1d, middle). C. elegans attraction increased for $24 \mathrm{~h}$ and remained high even after $72 \mathrm{~h}$, similar to the trend observed in diacetyl production (Figure 1d, bottom). Thus, C. elegans attraction to $L$. paracasei grown on citrate media correlates with diacetyl production, rather than bacterial growth patterns.

\section{Adaptation to diacetyl inhibits attraction to $\mathrm{L}$. paracasei grown on citrate}

Is $C$. elegans actually able to distinguish between diacetyl-producing and non-producing $L$. paracasei? One way to test this is to take advantage of the ability of $C$. elegans to adapt to the odor diacetyl. Worms are strongly attracted to diacetyl, but after about $1 \mathrm{~h}$ pre-exposure to diacetyl, worms will adapt to the odor and ignore it (Colbert and Bargmann, 1995). We therefore pre-exposed worms to diacetyl for $80 \mathrm{~min}$, and then assessed whether attraction to LAB grown on citrate media was attenuated. As expected, worms pre-exposed to diacetyl no longer showed attraction to diacetyl on an odortaxis assay. Interestingly, adaptation to diacetyl also resulted in the disappearance of attraction to LAB grown on citrate (Figure 2a).

Attractive odors are sensed by two pairs of sensory neurons at the anterior of $C$. elegans, called the AWA and AWC neuron pairs (Bargmann et al., 1993). The AWA neurons are responsible for the attraction to diacetyl, whereas the AWC neurons sense other odors such as benzaldehyde, a chemical with an almond scent. Adaptation to benzaldehyde is also mediated by the AWC neurons. Thus, adaptation to benzaldehyde is completely independent of adaptation to diacetyl. To test whether the attenuation of attraction to LAB/citrate was specific for the diacetyl odor adaptation, we tested whether benzaldehyde pre-exposure could affect LAB/citrate attraction. As shown in Figure 2a, benzaldehyde pre-exposure resulted in adaptation behavior to benzaldehyde odortaxis. However, benzaldehyde pre-exposure had no effect on attraction to LAB grown on citrate. Thus, the adaptation response to $L$. paracasei was specific for the odor diacetyl. This strongly suggests that attraction to $L$. paracasei in $C$. elegans is mediated by diacetyl production in LAB.

\section{The diacetyl receptor ODR-10 mediates attraction} to L. paracasei

The adaptation experiments indicate a behavioral specificity for attraction to Lactobacillus that 
produce diacetyl. We sought to provide molecular and genetic specificity for attraction to Lactobacillus. From mammals to nematodes, odors are sensed by membrane-bound G-protein coupled receptors (GPCRs). There are over a thousand GPCR-encoding genes in the C. elegans genome. However, in seminal work from the Bargmann lab, the odor receptor responsible for sensing diacetyl was identified as ODR-10 (Sengupta et al., 1996). Mutants of odr-10 are not attracted to diacetyl. We asked whether ODR-10 was also mediating the attraction to
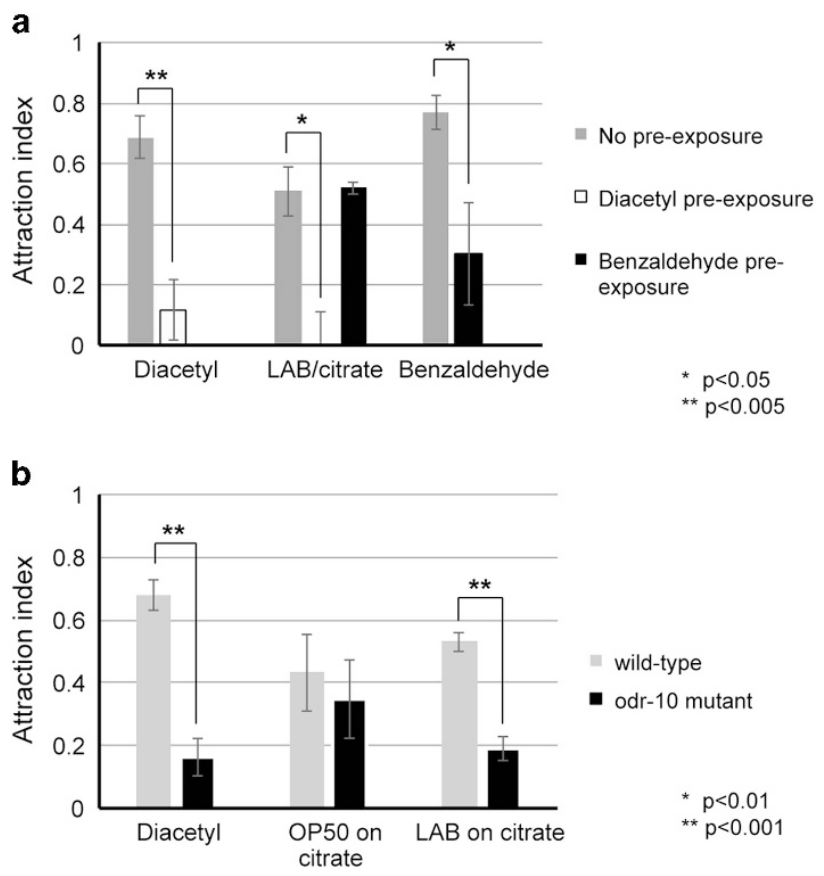

Figure 2 C. elegans is attracted to diacetyl produced by LAB grown on citrate media. (a) Pre-exposure to the odor diacetyl results in adaptation behavior towards LAB grown on citrate media. Wild-type $C$. elegans was pre-exposed to 1:5000 dilution diacetyl, 1:5000 dilution benzaldehyde or a control buffer for $80 \mathrm{~min}$, then performed an attraction assay either to diacetyl, LAB grown on citrate media or benzaldehyde. Error bars indicate s.e. Significance was calculated by Student's $t$-test and $P$-values are indicated. (b) Attraction was then tested to diacetyl, OP50 on citrate media, or LAB on citrate media with wild-type $C$. elegans and odr-10(ky32) mutants carrying a mutation in the ODR-10 diacetyl receptor. Error bars indicate s.e. Significance was calculated by Student's $t$-test and $P$-values are indicated.
L. paracasei grown on citrate media. In odr-10(ky10) mutants, attraction to diacetyl was significantly lower than wild-type $\mathrm{N} 2$ animals (Figure $2 \mathrm{~b}$ ). Interestingly, we saw a similar loss of attraction to Lactobacillus on the citrate media in odr-10 mutants (Figure 2b). Thus, these experiments provide behavioral, genetic and molecular evidence that $C$. elegans is attracted to diacetyl produced by L. paracasei.

\section{Both Caenorhabditis nematodes and LABs inhabit rotting fruit in nature}

The most obvious source of citrate in nature is in fruits. Coincidently, one of the common places to find $C$. elegans and other nematodes is within rotting fruits (Felix and Braendle, 2010; Felix and Duveau, 2012). We wanted to confirm this previous finding locally in the Korean peninsula. We collected rotten fruit and adjacent soil from several fruit farms during each fruit harvest season. In the laboratory, we picked out nematodes from within these rotten fruit samples, and identified the nematodes by $18 \mathrm{~S}$ rDNA sequencing. We successfully found Caenorhabiditis species such as $C$. remanei and $C$. briggsae in pear and yuzu fruit samples (Table 1). We also found other types of nematodes such as Panagrellus and Pristionchus that were also previously reported to be found in fruit (Felix and Duveau, 2012).

LABs are fermentative bacteria that are well known to ferment many types of foods including fruits and vegetables. We asked whether any LABs were among the species of bacteria residing in our rotten fruit samples. Thus, we isolated bacteria from our rotten fruit samples and identified LAB strains by $16 \mathrm{~S}$ rDNA sequencing. From these samples we found members of the genera Lactobacillus and Lactococcus bacteria in rotten apple, pear, peach and yuzu fruit (Table 1). Taken together, we conclude that LABs and Caenorhabditis nematodes co-inhabit rotting fruit.

\section{C. elegans is attracted to Lactobacillus fermenting citrus} fruit media

It was already known that adult $C$. elegans, as well as other nematodes, could be found in relatively large populations in and near rotting fruits (Felix and Braendle, 2010; Kiontke et al., 2011). However, it is

Table 1 Nematodes and LAB isolated and identified from rotten fruit

\begin{tabular}{|c|c|c|c|c|}
\hline Fruit & Location & Date & $L A B$ identified & Nematode identified \\
\hline Apple & Chungju, S. Korea & Oct 2013 & Lactobacillus sp. AS & Not sampled \\
\hline Pear & Wonju, S. Korea & Oct 2013 & Lactobacillus sp. PS03 Lactobacillus sp. PS04 & Not sampled \\
\hline Peach & Wonju, S. Korea & Aug 2014 & Lactobacillus sp. D4 Lactobacillus sp. D5 & Not sampled \\
\hline Pear & Wonju, S. Korea & Oct 2014 & $\begin{array}{l}\text { Lactobacillus sp. PC04 Lactococcus lactis PB1 } \\
\text { Lactobacillus plantarum PB2 }\end{array}$ & $\begin{array}{l}\text { Pristionchus sp. Panagrellus sp. C. briggsae } \\
\text { strain PC01 }\end{array}$ \\
\hline Yuzu & Goheung, S. Korea & Nov 2014 & $\begin{array}{l}\text { Lactobacillus sp. Y091 Lactobacillus sp. Y092 } \\
\text { Lactococcus lactis YS } 252\end{array}$ & C. remanei strain Y01 Pelodera sp. \\
\hline
\end{tabular}

Rotten apple, peach, pear and yuzu were sampled from four locations. Pear was sampled once in 2013 and once in 2014. Genus or species identification is based on partial sequencing of $16 \mathrm{~S}$ or 18S rDNA genomic sequencing. 
not necessarily known why $C$. elegans prefers to reside in rotting fruit, or how they arrived there. Caenorhabditis and closely related nematodes are not likely to draw much nutritional benefit from nonmicrobial matter (Houthoofd et al., 2002). One possibility is that $C$. elegans may be attracted to rotting fruit by the abundant and diverse populations of bacteria that ferment and use the sugars in fruit. Caenorhabditis and closely related nematodes are known as enrichment-type nematodes that grow and reproduce quickly to enormous numbers in the presence of quality bacterial food sources. We hypothesized, then, that the diacetyl attraction behavior we observed was actually a natural ecological behavior for $C$. elegans to find a large and growing source of bacterial food, sort of a 'bacterial bonanza', and that diacetyl acts as a cue for worms to track down the bacterial bonanza.

To investigate this hypothesis, we needed to find out whether LABs could produce diacetyl while fermenting citrate-containing fruits. To do this, we made several types of agar media using different fruits and a grain vegetable, a relatively common technique to culture LAB (Elliker and Hannesson, 1956). We used three different fruit-based media: yuzu, tomato and apple. Yuzu fruit (Citrus medica L.) is a citrus fruit native to East Asia that contains very high levels of citrate, and is grown agriculturally on the southern tip of South Korea. Tomato, although not a citrus fruit, contains some citrate. Apple is an acidic fruit that contains high levels of malic acid, but low levels of citrate. As a negative control, we used barley malt that contains little or no citrate. We grew L. paracasei on each of these fruit/vegetable media plates for 2 days, and then measured diacetyl production by spectrophotometric analysis. As seen in Figure 3a, LAB grown on the tomato, apple and barley media produce little diacetyl. In contrast, LAB grown on the yuzu media produces considerable levels of diacetyl albeit less than on citrate media.

We then assayed $C$. elegans attraction to LAB grown on each of the fruit/vegetable media. L. paracasei grown on barley malt media produces little diacetyl (Figure 3a). We found, though, that C. elegans is highly attracted to LAB on barley malt media (Figure $3 \mathrm{~b}$ ). However, this attraction is not dependent on the diacetyl receptor, as odr-10 mutants showed significant attraction to barley-grown LAB (Figure 3b, black bars). Thus, other odors may be mediating this attraction. Yuzu fruit, on the other hand, contains high levels of citrate, and L. paracasei grown on yuzu media produces high levels of diacetyl (Figure 3a). When we tested attraction of wild-type worms to LAB grown on yuzu, we found that it was highly attractive, but strikingly, very little attraction was seen in the odr-10 mutants, indicating that this attraction was mediated by the diacetyl receptor. Growth on apple and tomato media results in low or moderate levels of diacetyl. Consistent with this,

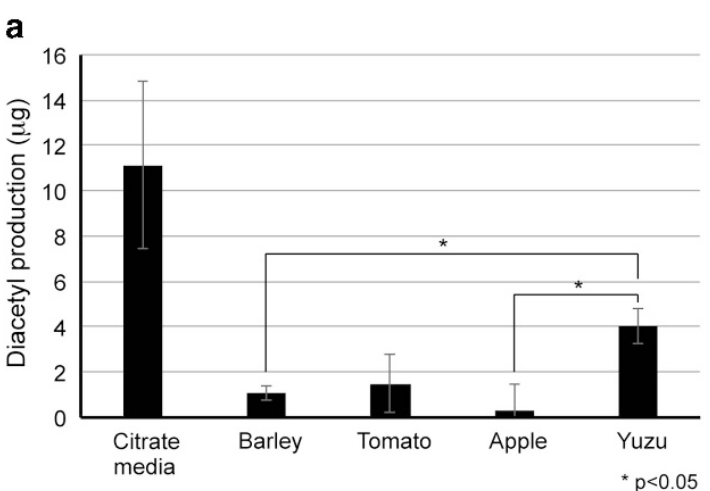

b

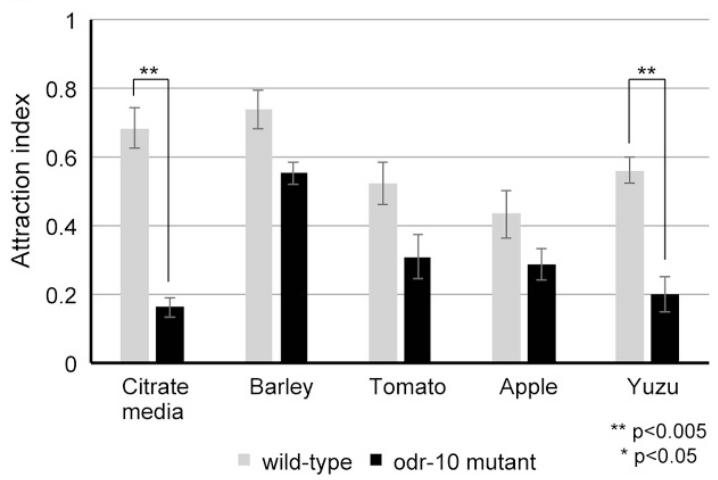

Figure 3 C. elegans is attracted to diacetyl produced by LAB grown on media made from the citrus fruit yuzu. LAB was grown for 2 days on media made from apple, tomato, barley malt, or yuzu, and diacetyl production (a) or attraction behavior of wildtype and odr-10 mutants (b) of an agar plug from the plates was assessed. Diacetyl standard curves (Supplementary Figure S2) were used to calculate diacetyl levels. Error bars indicate s.e. Significance was calculated by Student's $t$-test and $P$-values are indicated.

both cause a moderate attraction to worms, and this is partially but not significantly mediated by ODR-10. Thus, diacetyl can mediate attraction to Lactobacillus that are fermenting citrus fruit media.

Wild Caenorhabditis is attracted to wild LAB strains isolated from yuzu

We have demonstrated that both Rhabditid nematodes and LAB can be found in fruits, and diacetyl produced by an LAB grown on citrate or citrus fruit media is attractive to $C$. elegans. However, whether this phenomenon of odor attraction between nematodes and LABs occurs in nature is not known. We wanted to test whether an LAB isolated from rotting fruits in nature produced diacetyl, and whether wild nematodes isolated from the same rotting fruit are attracted to the LAB. We first tested whether C. elegans was attracted to a wild LAB strain. We used the Lactobacillus strain Y091 that we isolated from yuzu fruit (Table 1), grew it for 2 days on citrate and yuzu media, and tested whether $C$. elegans was attracted to it. As shown in Figure 4a, wild-type C. elegans was attracted to the wild LAB grown on 


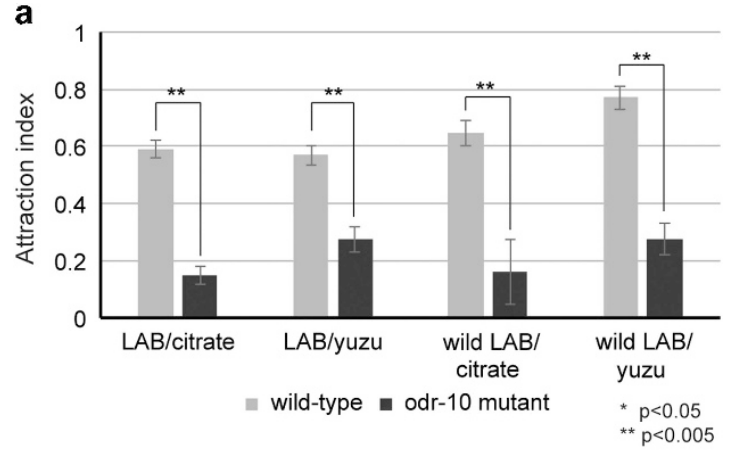

b

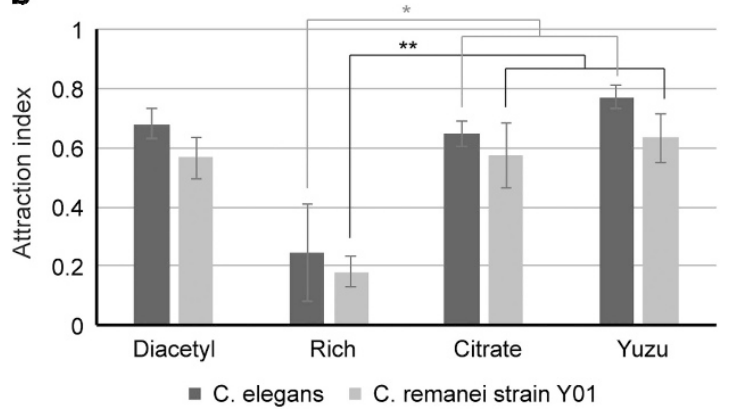

Figure 4 Wild Caenorhabditis strain is attracted to wild LAB strains isolated from yuzu. (a) Attraction behavior of wild-type and odr-10 mutant C. elegans to wild Lactobacillus strain Y091 isolated from rotten yuzu was assessed, when grown on either citrate media or yuzu media. (b) Attraction behavior of wild-type C. elegans and wild C. remanei strain Y01 isolated from rotten yuzu to wild Lactobacillus strain Y091 was assessed. Wild LAB was grown on either citrate or yuzu media. Error bars indicate s.e. Significance was calculated by Student's $t$-test and $P$-values are indicated.

either media. To test whether this attraction was mediated by diacetyl sensation, we tested odr-10 mutants for attraction behavior. Worms that lack the diacetyl receptor were no longer attracted to the wild LAB grown on either citrate or yuzu media. Thus, C. elegans attraction to a wild LAB strain isolated from yuzu is mediated by diacetyl sensation.

All nematodes including C. elegans use their sense of smell to track cues in the environment. In fact, many other nematodes including the closely related Rhabditid nematode Caenorhabditis remanei is attracted to the odor diacetyl (Hong and Sommer, 2006). We isolated a wild strain of $C$. remanei from rotten yuzu (Table 1), and tested its attraction to diacetyl. As shown in Figure 4b, this wild strain was attracted to diacetyl, similarly to the laboratory strain of C. remanei (Hong and Sommer, 2006). Next, we asked whether this wild $C$. remanei was attracted to the wild LAB strain Y091 that was also isolated from yuzu fruit. Y091 wild LAB grown on either citrate or yuzu was highly attractive to wild $C$. remanei isolate from yuzu, but not attractive when grown on rich media (Figure 4b). Taken together, these data support the hypothesis that nematodes sense diacetyl produced by LAB growing on citrus fruit media.

\section{Discussion}

LAB have been well studied due to their importance in the food industry and the probiotic benefits they confer towards human health. However, little is known about the ecology of LABs in nature outside of the human gut. Likewise, C. elegans has been one of the most useful laboratory genetic model organisms over the last 40 years, but little is known about natural interactions with $C$. elegans in nature. Here, we employed diverse biological methods and attempted to quantify a natural ecological phenomenonon in the laboratory using $L$. paracasei and $C$. elegans genetics. We showed that $C$. elegans is attracted to L. paracasei grown on citrate or yuzu media. We demonstrated that the attraction to the LAB arises specifically from diacetyl odor signaling. This phenomenon was not limited to $C$. elegans and L. paracasei, but wild Caenorhabditis isolated from rotten yuzu fruit also shows a robust attraction to Lactobacillus isolated from rotten yuzu fruit grown on citrate media or yuzu media. We have described a genetic and molecular basis for an ecological interaction between bacteriovorous nematodes and bacteria in rotten fruit. These experiments also establish a natural role for odor behaviors and sensory circuitry in an animal.

Our hypotheses are that (1) first, LAB grows on fresh citrus fruit, (2) LAB metabolize sugar utilizing citrate in the fruit, (3) diacetyl is produced as a byproduct, (4) nematodes sense diacetyl, activating diacetyl odor receptors, (5) nematodes are attracted to diacetyl and navigate to the source of LAB, (6) nematodes find a diverse bacterial bonanza, lay eggs and quickly reproduce.

The study of olfactory circuitry and odor behaviors in an animal with a simple and intact nervous system like $C$. elegans has been invaluable in understanding odor discrimination, odor memory and adaptation, and integration with other sensory systems. However, this study is the first demonstration to our knowledge of a natural odor attraction between $C$. elegans and a specific odor released by bacteria found in the nematode's environment. In addition, we show that after pre-exposing the worms to chemical diacetyl without bacteria, C. elegans adapts to the odor and ignores the diacetyl emanating from the LAB during the attraction assay. It is thought that in nature odor adaptation is a behavioral mechanism for worms to ignore profitless odors and focus on odors that lead the worm to food. Diacetyl production is long-range volatile cue that may lead the worms to the area of LAB growth, but LAB itself may not be a preferable source of nutrition for C. elegans. This could lead to adaptation to diacetyl. One speculation is that adaptation to diacetyl may help focus the worm on searching for nearby sources of preferred bacteria. Overall, these experiments attribute ecological significance for odor attraction behavior and memory in nematodes. 
We speculate that many other odors emanate from countless other microorganisms that they interact with and may account for the large number of chemosensory receptors that have evolved within the Caenorhabditis genomes (Robertson, 1998; Thomas and Robertson, 2008). In fact, we observe diacetyl receptor-independent attraction to LAB grown on barley, tomato and apple, indicating odors other than diacetyl are mediating attraction in these assays. Furthermore, a recent investigation has suggested the importance of the diacetyl receptor for $C$. elegans to sense E. coli bacteria, allowing males to make correct behavioral choices between eating and mating (Ryan et al., 2014). Attraction to diacetyl odor in C. remanei and the closely related nematode Caenorhabditis briggsae has been established (Hong and Sommer, 2006). In this study, we demonstrate that wild $C$. remanei isolated from rotten yuzu is attracted to LAB grown on citrate or yuzu media. Whether a homolog of ODR-10 in C. remanei mediates this attraction requires further study.

Although we demonstrated an attraction to diacetyl emanating from a LAB in rotten fruit by nematodes in laboratory conditions, whether diacetyl sensation allows nematodes to increase nutrition and acquire any reproductive fitness advantages requires a broader study. In fact, our observations confirm other studies that show C. elegans grown solely on Lactobacillus develop slowly and become calorically restricted (Kim and Mylonakis, 2012; Zhao et al., 2013). However, LABs are only one type of bacteria out of many that reside in rotten fruit. In further experiments, we identified two soil bacteria, Enterobacter sp. and Arthrobacter sp., in the intestines of wild $C$. remanei strain Y01 that we recovered from rotten yuzu fruit (data not shown). In addition, we found that wild-type C. elegans produces moderate brood sizes when grown on Arthrobacter sp. and large brood sizes when grown on Enterobacter sp. comparable to the large brood sizes observed when grown on laboratory strain OP50 E. coli (data not shown). This shows that nematodes can find and consume various bacteria in fruit. Previous studies report that increased numbers of nematodes are found in areas of the soil that are populated with increased diversity of bacteria (Bongers, 1990; Darby et al., 2007). Our hypothesis is that diacetyl acts as a cue to allow nematodes to locate large and diverse bacterial bonanzas growing in an area where nutrients for microbial growth are abundant.

The molecular and genetic studies of C. elegans and the microbiological and biochemical studies of LAB have contributed greatly to our understanding of basic biology and human health. Our work takes advantage of the breadth of knowledge accumulated in $C$. elegans and $\mathrm{LAB}$ research to identify how animals and microbes may interact in nature at the molecular level. Further study should elucidate other molecules that mediate ecological interactions between C. elegans and LAB and other microbial communities.

\section{Conflict of Interest}

The authors declare no conflict of interest.

\section{Acknowledgements}

We thank Se Wook Farms (Wonju), Chiaksan Peach Farms (Wonju) and Ja So Dahm Farms (Goheung) for allowing us to collect fruit samples, the Caenorhabditis Genetic Center for strains, Damien O'Halloran and Noelle L'Etoile for critical reading of the manuscript, and members of the Lee and Yoon labs. This work was supported by a Yonsei University Challenge Grant (2014-22-0152) and a New Investigator Grant from the Korea National Research Foundation (2015-51-0104).

\section{References}

Aunsbjerg SD, Honore AH, Marcussen J, Ebrahimi P, Vogensen FK, Benfeldt C et al. (2015). Contribution of volatiles to the antifungal effect of Lactobacillus paracasei in defined medium and yogurt. Int J Food Microbiol 194: 46-53.

Bargmann CI, Horvitz HR. (1991). Chemosensory neurons with overlapping functions direct chemotaxis to multiple chemicals in C. elegans. Neuron 7: 729-742.

Bargmann CI, Hartwieg E, Horvitz HR. (1993). Odorant-selective genes and neurons mediate olfaction in C. elegans. Cell 74: 515-527.

Barriere A, Felix MA. (2005). High local genetic diversity and low outcrossing rate in Caenorhabditis elegans natural populations. Curr Biol 15: 1176-1184.

Barriere A, Felix MA. (2014). Isolation of C. elegans and related nematodes. WormBook; e-pub ahead of print 2 May 2014; doi:10.1895/wormbook.1.115.2.

Bartowsky EJ, Henschke PA. (2004). The 'buttery' attribute of wine-diacetyl-desirability, spoilage and beyond. Int J Food Microbiol 96: 235-252.

Bongers T. (1990). The maturity index - an ecological measure of environmental disturbance based on nematode species composition. Oecologia 83: 14-19.

Buck L, Axel R. (1991). A novel multigene family may encode odorant receptors: a molecular basis for odor recognition. Cell 65: 175-187.

Colbert HA, Bargmann CI. (1995). Odorant-specific adaptation pathways generate olfactory plasticity in C. elegans. Neuron 14: 803-812.

Darby BJ, Neher DA, Belnap J. (2007). Soil nematode communities are ecologically more mature beneath late- than early-successional stage biological soil crusts. Appl Soil Ecol 35: 203-212.

Dusenbery DB. (1975). The avoidance of D-tryptophan by the nematode Caenorhabditis elegans. J Exp Zool 193: 413-418.

Elliker PRA, Hannesson AW. (1956). An agar culture medium for lactic acid Streptococci and Lactobacilli. J Dairy Sci 39: 1611-1612.

Felix MA, Braendle C. (2010). The natural history of Caenorhabditis elegans. Curr Biol 20: R965-R969. 
Felix MA, Duveau F. (2012). Population dynamics and habitat sharing of natural populations of Caenorhabditis elegans and C. briggsae. BMC Biol 10: 59.

Ferrero DM, Lemon JK, Fluegge D, Pashkovski SL, Korzan WJ, Datta SR et al. (2011). Detection and avoidance of a carnivore odor by prey. Proc Natl Acad Sci USA 108: 11235-11240.

Floyd R, Abebe E, Papert A, Blaxter M. (2002). Molecular barcodes for soil nematode identification. Mol Ecol 11: 839-850.

Gray JM, Hill JJ, Bargmann CI. (2005). A circuit for navigation in Caenorhabditis elegans. Proc Natl Acad Sci USA 102: 3184-3191.

Guarner F, Malagelada JR. (2003). Gut flora in health and disease. Lancet 361: 512-519.

Hammer BW. (1920). The type of lactic acid produced by starters and by the organisms isolated from them. Iowa State Coll Agr Exp Station Res Bull 65: 115-128.

Harris G, Shen Y, Ha H, Donato A, Wallis S, Zhang X et al. (2014). Dissecting the signaling mechanisms underlying recognition and preference of food odors. J Neurosci 34: 9389-9403.

Hedgecock EM, Russell RL. (1975). Normal and mutant thermotaxis in the nematode Caenorhabditis elegans. Proc Natl Acad Sci USA 72: 4061-4065.

Hempel S, Newberry SJ, Maher AR, Wang Z, Miles JN, Shanman R et al. (2012). Probiotics for the prevention and treatment of antibiotic-associated diarrhea: a systematic review and meta-analysis. JAMA 307: 1959-1969.

Hills T, Brockie PJ, Maricq AV. (2004). Dopamine and glutamate control area-restricted search behavior in Caenorhabditis elegans. J Neurosci 24: 1217-1225.

Hong RL, Sommer RJ. (2006). Chemoattraction in Pristionchus nematodes and implications for insect recognition. Curr Biol 16: 2359-2365.

Houthoofd K, Braeckman BP, Lenaerts I, Brys K, De Vreese A, Van Eygen S et al. (2002). Axenic growth up-regulates mass-specific metabolic rate, stress resistance, and extends life span in Caenorhabditis elegans. Exp Gerontol 37: 1371-1378.

Hugenholtz J. (1993). Citrate metabolism in lactic acid bacteria. FEMS Microbiol Rev 12: 165-178.

Ikeda T, Yasui C, Hoshino K, Arikawa K, Nishikawa Y. (2007). Influence of lactic acid bacteria on longevity of Caenorhabditis elegans and host defense against salmonella enterica serovar enteritidis. Appl Environ Microbiol 73: 6404-6409.

Jyoti BD, Suresh AK, Venkatesh KV. (2004). Effect of preculturing conditions on growth of Lactobacillus rhamnosus on medium containing glucose and citrate. Microbiol Res 159: 35-42.

Kim Y, Mylonakis E. (2012). Caenorhabditis elegans immune conditioning with the probiotic bacterium Lactobacillus acidophilus strain NCFM enhances gram-positive immune responses. Infect Immun 80: 2500-2508.

Kiontke KC, Felix MA, Ailion M, Rockman MV, Braendle C, Penigault JB et al. (2011). A phylogeny and molecular barcodes for Caenorhabditis, with numerous new species from rotting fruits. BMC Evol Biol 11: 339.
Lewis JA, Fleming JT. (1995). Basic culture methods. In: Epstein HF, Shakes DC (eds). Caenorhabditis elegans: Modern Biological Analysis of an Organism. Academic Press, Inc.: San Diego, CA, USA, pp 4-27.

Liu KS, Sternberg PW. (1995). Sensory regulation of male mating behavior in Caenorhabditis elegans. Neuron 14: 79-89.

Loer CM, Kenyon CJ (1993). Serotonin-deficient mutants and male mating behavior in the nematode Caenorhabditis elegans. J Neurosci 13: 5407-5417.

Marshall VM. (1987). Lactic acid bacteria: starters for flavour. FEMS Microbiol Rev 3: 327-336.

Mattessich J, Cooper JR. (1989). The spectrophotometric determination of diacetyl. Anal Biochem 180: 349-350.

Michaelian MB, Hoecker WH, Hammer BW. (1938). Effect of $\mathrm{pH}$ on the production of acetylmethylcarbinol plus diacetyl in milk by the citric acid fermenting Streptococci. J Dairy Sci 21: 213-218.

Michaelian MB, Hammer BW. (1933). The relationship of acetylmethylcarbinol and diacetyl to butter cultures. Iowa State Coll Agr Exp Station Res Bull 155: 204-227.

Papes F, Logan DW, Stowers L. (2010). The vomeronasal organ mediates interspecies defensive behaviors through detection of protein pheromone homologs. Cell 141: 692-703.

Robertson HM. (1998). Two large families of chemoreceptor genes in the nematodes Caenorhabditis elegans and Caenorhabditis briggsae reveal extensive gene duplication, diversification, movement, and intron loss. Genome Res 8: 449-463.

Ryan DA, Miller RM, Lee K, Neal SJ, Fagan KA, Sengupta P et al. (2014). Sex, age, and hunger regulate behavioral prioritization through dynamic modulation of chemoreceptor expression. Curr Biol 24: 2509-2517.

Sengupta P, Chou JH, Bargmann CI. (1996). odr-10 encodes a seven transmembrane domain olfactory receptor required for responses to the odorant diacetyl. Cell $\mathbf{8 4}$ : 899-909.

Smit G, Smit BA, Engels WJ. (2005). Flavour formation by lactic acid bacteria and biochemical flavour profiling of cheese products. FEMS Microbiol Rev 29: 591-610.

Song TL, Kang K., Yoo S, Lee S, Yoon SS. (2013). Synthesis of Galactooligosaccharides in the Cheese Whey-based Medium by a Lactase from Lactobacillus paracasei YSM0308. Korean J Food Sci An 33: 565-571.

Thomas JH, Robertson HM. (2008). The Caenorhabditis chemoreceptor gene families. BMC biology 6: 42 .

Trias R, Baneras L, Montesinos E, Badosa E. (2008). Lactic acid bacteria from fresh fruit and vegetables as biocontrol agents of phytopathogenic bacteria and fungi. Int Microbiol 11: 231-236.

Ward S. (1973). Chemotaxis by the nematode Caenorhabditis elegans: identification of attractants and analysis of the response by use of mutants. Proc Natl Acad Sci USA 70: 817-821.

Zhao Y, Zhao L, Zheng X, Fu T, Guo H, Ren F. (2013). Lactobacillus salivarius strain FDB89 induced longevity in Caenorhabditis elegans by dietary restriction. J Microbiol 51: 183-188.

Supplementary Information accompanies this paper on The ISME Journal website (http://www.nature.com/ismej) 